\title{
Cross-linguistic evidence for cognitive universals in the noun phrase
}

\author{
Alexander Martin, Theeraporn Ratitamkul, Klaus Abels, David Adger, \& Jennifer Culbertson
}

\section{Introduction}

Apparent regularities across languages, known as typological generalisations, have fueled major debates in linguistics for decades (e.g., see Evans \& Levinson 2009, and commentaries). This paper concerns two generalisations in the neutral word order of the noun phrase. We report two experiments which are part of a larger research programme intended to support experimentally the idea that distributional regularities in noun phrase order result from preferences or constraints operating on linguistic representations in individual human minds. Concretely, our starting point is a generalisation known as Universal 20, first proposed by Greenberg (1963):

"In pre-nominal position the order of demonstrative, numeral, and adjective (or any subset thereof) conforms to the order Dem-Num-Adj. In post-nominal position the order of the same elements (or any subset thereof) conforms to either the order Dem-Num-Adj or to the order Adj-Num-Dem." (as re-stated in Cinque 2005).

While there are twenty-four possible orderings of these four elements, current typological samples reveal that two patterns dominate, accounting for nearly 50\% of all languages (Cysouw 2010). One is the order found in English: Dem Num Adj N. The other is its mirror: N Adj Num Dem (found, for example, in Thai). The latter is the most common. A number of other patterns are less frequent, but robustly attested. After that follows a long tail of infrequently attested orders, and some orders which are not known to be used as the dominant (or neutral) pattern in any language (e.g., Adj Dem N Num, see Fig. 1).

Several prominent explanations for this typological generalisation are based on claims about universal underlying features of noun phrase structure or semantics (Abels \& Neeleman 2012; Cinque 2005; Dryer 2018; Steddy \& Samek-Lodovici 2011; Steedman 2018). These proposals start from the observation that adjectives tend to be closer to the nouns they modify than numerals, which in turn are closer to the noun than demonstratives. If we cash out closeness in terms of phrase structure, adjectives are grouped with nouns to the exclusion of numerals and numerals with adjective-noun combinations to the exclusion of demonstratives. Culbertson and Adger (2014) suggest that these structures are motivated by considerations of semantic scope. Adjectives combine semantically with the noun, forming a constituent (more precisely, a nominal predicate). Numerals combine with these constituents to form countable units. Finally, demonstratives map these (sub-)constituents to individuals. Demonstratives therefore scope over both numerals and adjectives, numerals scope over adjectives, and adjectives do not (generally) scope over either of these two. Put another way, adjectives are closer to nouns because they reflect properties inherent to the noun, while demonstrative are 
furthest away because they relate material internal to the noun phrase to external information like discourse status, spatial location relative to the speaker, etc.

Logically, these semantic relations need not determine linear order. That is, we can imagine arbitrary relationships between order and semantic scope, learned from exposure to surface order in a language. This would predict a random distribution of language types, contra Fig. 1.

Alternatively, we can imagine a more restricted relationship, where order and scope are isomorphic (Culbertson \& Adger 2014). Isomorphism essentially swaps a linear relation between two elements for the semantic scope relation: if A semantically scopes over B, then A linearly precedes/follows B and everything contained within B. The eight isomorphic linear orderings of the semantic scope relations are shown in (1).

(1) a.

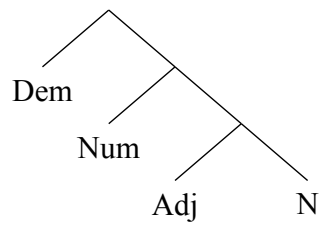

c.

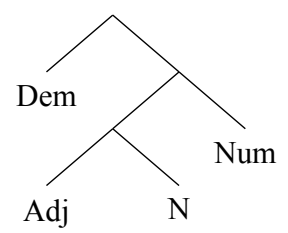

e.

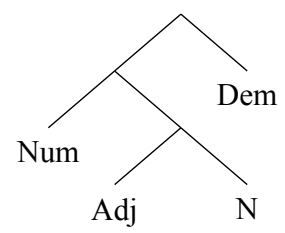

g.

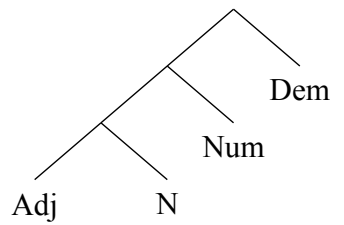

b.

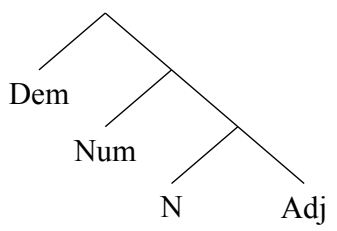

d.

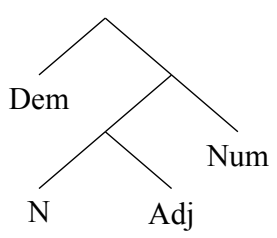

f.

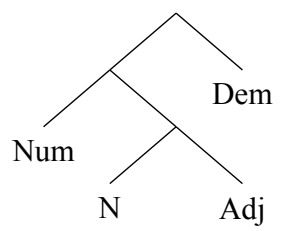

h.

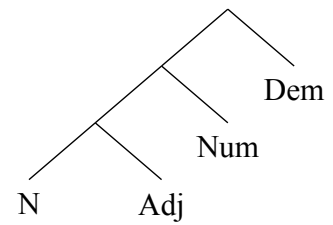

Each of these eight orders corresponds to a phrase structure tree which obeys the hierarchy above and in which branches are not tangled. These orders result from a binary decision, for each (sub-)constituent, concerning the linear order of the elements in that constituent. For example, if $\mathrm{N}$ preceded Adj linearly, and the constituent which includes [N Adj] precedes Num, and the constituent which includes [[N Adj] Num] precedes Dem, the linear order will be N Adj Num Dem. Crucially, in all isomorphic orders, the adjective is closest to the noun linearly, and the demonstrative is furthest 

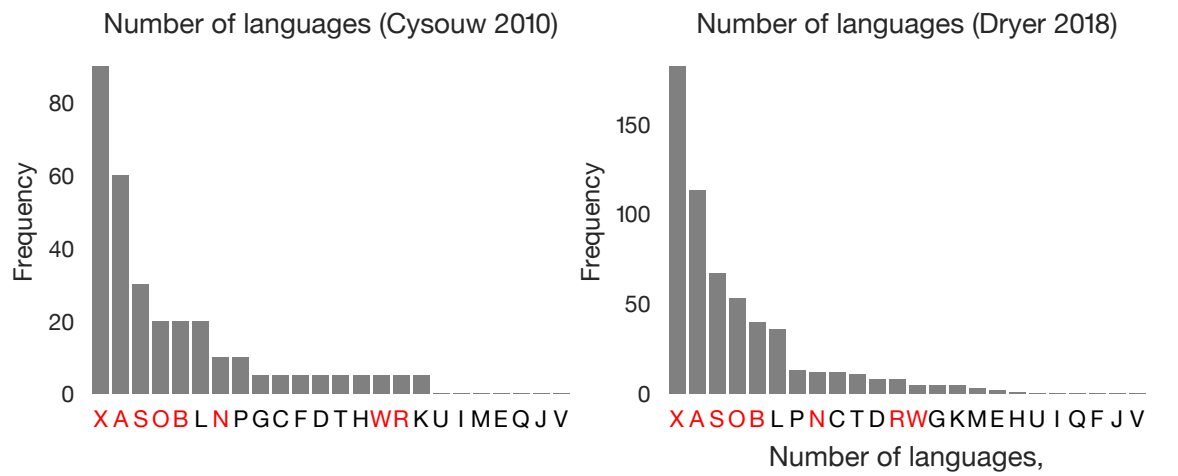

A: Dem-Num-A-N

B: Dem-Num-N-A

C: Dem-N-Num-A

D: N-Dem-Num-A

E: Num-Dem-A-N

F: Num-Dem-N-A

G: Num-N-Dem-A

$\mathrm{H}: \mathrm{N}-\mathrm{Num}-\mathrm{Dem}-\mathrm{A}$

I: A-Dem-Num-N

$\mathrm{J}$ : A-Dem-N-Num

$\mathrm{K}$ : A-N-Dem-Num

L: N-A-Dem-Num

$\mathrm{M}$ : Dem-A-Num-N

$\mathrm{N}$ : Dem-A-N-Num adjusted for genetic relations (Dryer 2018)

O: Dem-N-A-Num
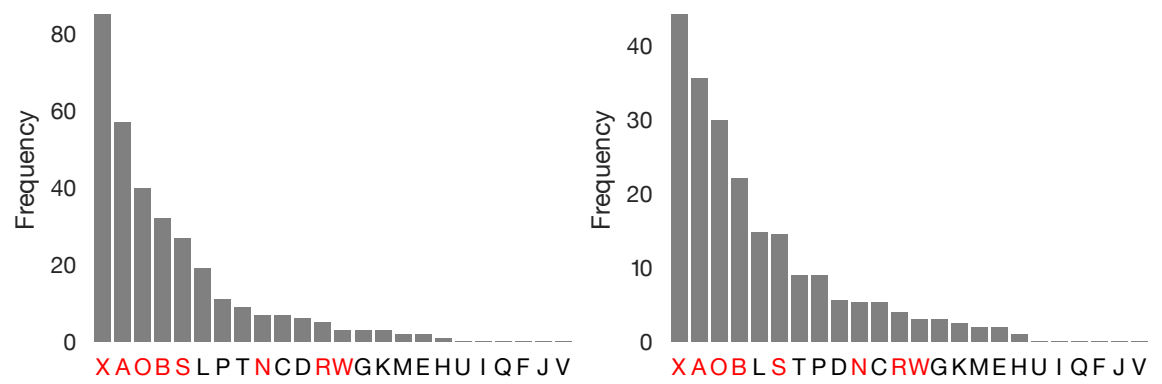

P: N-Dem-A-Num

Q: Num-A-Dem-N

R: Num-A-N-Dem

S: Num-N-A-Dem

T: N-Num-A-Dem

$\mathrm{U}$ : A-Num-Dem-N

$\mathrm{V}: \mathrm{A}-\mathrm{Num}-\mathrm{N}-\mathrm{Dem}$

$\mathrm{W}$ : A-N-Num-Dem

$\mathrm{X}$ : N-A-Num-Dem

Figure 1: Frequency counts of the twenty-four possible orderings of the noun phrase as reported by Cysouw (2010) and Dryer (2018). Orders in red are isomorphic; the letter codes correspond to those used by Cinque (2005).

away. An order like Adj Dem N Num is non-isomorphic, and cannot be derived by simply choosing the linear order within each sub-constituent. To summarise, in isomorphic orders, semantic closeness is reflected in linear proximity ${ }^{1}$.

Returning to the typology, the two most common orders, N Adj Num Dem and Dem Num Adj $\mathrm{N}$, are both isomorphic. Moreover, all eight isomorphic orders are not only attested, but are among the most common orders according to a number of different cross-linguistic samples and sampling methods. ${ }^{2}$ This is illustrated in Fig. 1, where orders in red are isomorphic, and summarised in Fig. 2.

In addition to this asymmetry based on isomorphism, there is a further asymmetry, which is also foreshadowed in Greenberg's Universal 20: there appears to be more flexibility in order when modifiers follow the noun. Specifically, there are substantially more languages with non-isomorphic orders of post-nominal modifiers (e.g., N Dem Num Adj as in the Bantu language Kîitharaka, Muriungi 2008) than pre-nominal modifiers. In fact very few if any languages with pre-nominal nonisomorphic as the dominant (or neutral) order have been identified ${ }^{3}$. This is summarised in Fig. 3.

This asymmetry has also been built into some accounts of noun phrase word order typology

\footnotetext{
${ }^{1}$ This is not the only case in which semantic scope has been claimed to interact with linear order (e.g., see Baker 1985; Koopman \& Szabolcsi 2000; Rice 2000)

${ }^{2}$ The two most common orders, N Adj Num Dem and Dem Num Adj N, also have consistent order of modifiers, either all before or all after the noun. Consistent modifier order (or harmony) has been proposed as an explanation for the dominance of these two isomorphic patterns (Culbertson \& Adger 2014; Dryer 2018).

${ }^{3}$ Though, see Dryer (2018) and Nchare (2012) for discussion of possible counter-examples.
} 


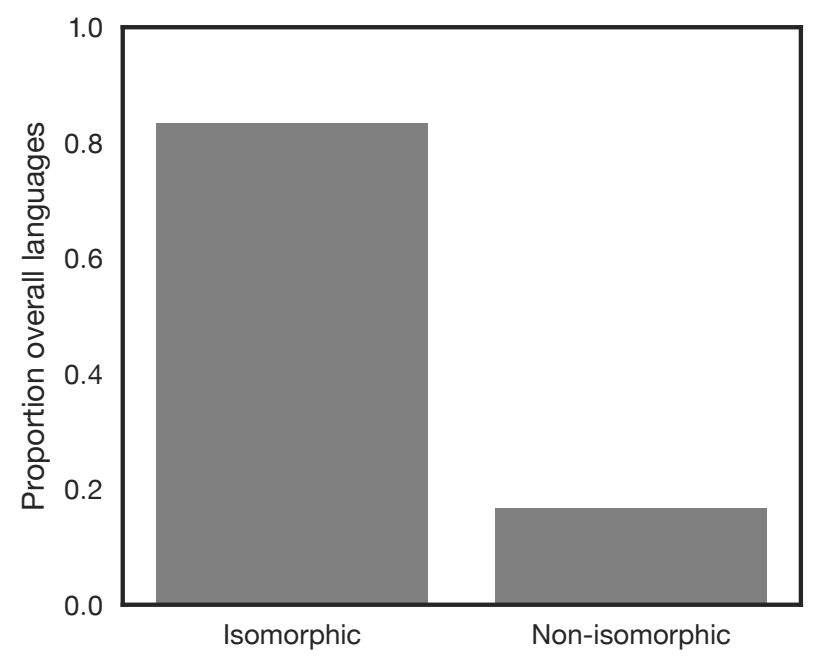

Figure 2: Proportion of languages defined as isomorphic and non-isomorphic, as reported in Cysouw (2010).

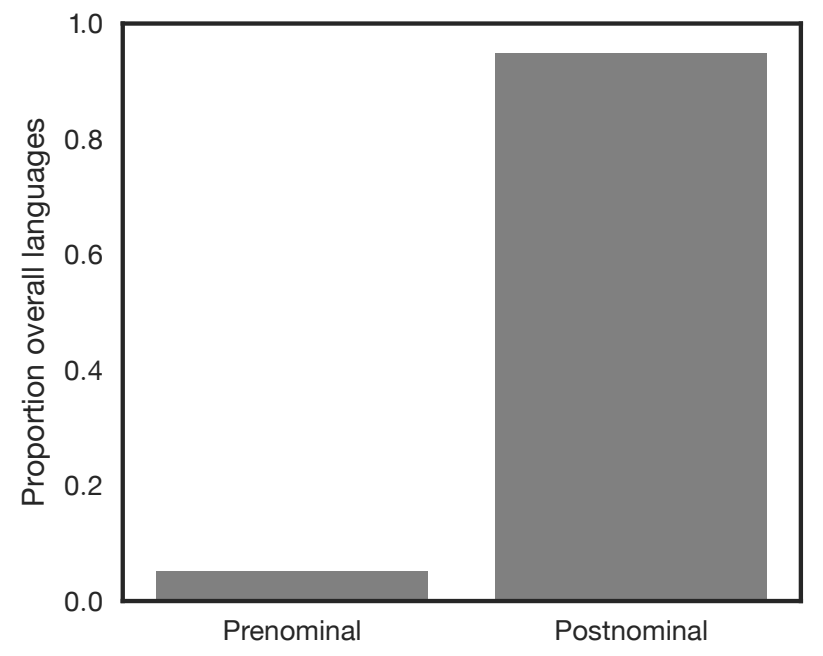

Figure 3: Proportion of non-isomorphic languages where the non-isomorphism occurs pre- and postnominally. Recall that this represents less than $20 \%$ of all languages. 
(Abels \& Neeleman 2012; Cinque 2005; Dryer 2018). Cinque (2005), for example, argues that all orders are derived from a structure which is linearised as Dem Num Adj N. Additional movement operations on that structure result in alternative orders. A ban on rightward movement derives the asymmetry among pre- and post-nominal orders, making pre-nominal non-isomorphism impossible. Abels and Neeleman (2012) achieve the same prohibition by positing that all orders must be derived from (one of) the eight isomorphic orders described above via leftward movement. Dryer (2018), argues instead that a preference for isomorphism (his "iconicity principle"), combined with a preference for post-nominal adjectives, makes pre-nominal non-isomorphic orders particularly unlikely.

What all of these accounts have in common is that they assume the typological patterns we see in Fig. 1 are the result of preferences or constraints operating on linguistic representations in individual human minds. First, they assume some representation of the underlying semantic relations among the elements in question. Second, they assume a preference or constraint which favours isomorphic linearisations in some way. Third, they assume an additional preference or constraint which targets pre-nominal orders specifically (rendering pre-nominal non-isomorphism rare or impossible to derive without special focus or contrastive meaning).

However, typology is only one source of evidence for these representations, and any preferences or constraints which operate on them. Even the relatively large current sample we havearound 700 languages-is still likely too small to allow us to draw strong conclusions about the nonaccidental nature of distributional asymmetries between particular patterns (Piantadosi \& Gibson 2014). Therefore, Culbertson and Adger (2014) sought out behavioural evidence to support these hypotheses, using an artificial language learning experiment. Culbertson and Adger (2014) taught English-speaking participants an artificial version of English where single-modifier noun phrases (e.g., "green car" or "this hat") began with the noun (thus, "car green" and "hat this"). During a test phase, when asked how this new version of English would translate two-modifier phrases such as "this green car", participants spontaneously assumed that the order of the two post-nominal modifiers would be isomorphic. That is, they overwhelmingly chose the order "car green this" over "car this green", and did so despite not receiving any input as to the relative order of modifiers during training. Importantly, in each case, participants chose the isomorphic order over a non-isomorphic alternative (N Dem Adj, N Dem Num, N Num Adj or N Dem Num Adj) that was demonstrably more likely given the surface order of English; modifier strings in these sequences are simply English linear order (just placed after the noun). Culbertson and Adger (2014) argue that English speakers are thus sensitive to an underlying representation of the noun phrase parallel to the one described above--in which the adjective is most closely related to the noun in terms of semantic constituency, and the demonstrative the least. This is consistent with the claim that such a structure is universal, and underlies the frequency of isomorphic patterns cross-linguistically. Of course, because English speakers could have inferred this structure from the word order in their language, it does not show this conclusively.

Here, we explore this effect further by replicating the basic result, and extending it to Thai speakers in order to target the second generalisation discussed above-the asymmetry between preand post-nominal isomorphism. In Thai, the order is isomorphic but the reverse of English. The accounts outlined above make a clear prediction: because pre-nominal orders show less variation in order than post-nominal ones, Thai speakers should have an even stronger preference for isomorphic orders pre-nominally than English speakers do post-nominally. To preview, we replicate the preference for isomorphism in both populations, but as predicted, Thai speakers show a stronger preference than English speakers. We discuss the possibility that this may be driven by second 
language knowledge of English.

\section{Experiment 1}

We first test the robustness of the effect reported in Culbertson and Adger (2014), namely that English speakers spontaneously assume isomorphic ordering of modifiers relative to the noun in a new language with post-nominal modifiers ${ }^{4}$.

\subsection{Methods}

Experiment 1 replicated Culbertson and Adger (2014), Experiment 1 with one main difference: our experiment was run in the lab, with University students, whereas the original experiment was run online (via Amazon Mechanical Turk). This and other changes were made so that both speaker populations could be tested under similar conditions.

\subsubsection{Stimuli}

The lexical items used were identical to the original experiment. They consisted of 30 nouns, 10 adjectives, 10 numerals, and four demonstratives ("this", "that", "these", "those"). All stimuli were recorded in a sound-attenuated room by a male speaker of British English whose accent was rhotic.

\subsubsection{Procedure}

The experiment consisted of two main phases: a training phase where participants were taught that modifiers in the new language followed the noun they modified, and a test phase where participants' preference for isomorphism was measured.

Participants were run in one of three experimental conditions. In the Dem-Adj condition, participants were exposed only to demonstratives and adjectives (in addition to nouns) in the new language; in the Dem-Num condition, only demonstratives and numerals; and in the Num-Adj condition, only numerals and adjectives.

Participants were told they would be learning to translate phrases into an English-like language. Written English noun phrases were displayed on screen (e.g., "green car") while audio was played of the new language. The new language differed from English only in that modifiers followed the noun. Thus, a participant might see "green car", but hear "car green". After audio presentation of the new language phrase, two buttons appeared on screen, one with the English phrase, and one with the new language phrase. The order of the two buttons was randomised across trials. Participants were instructed to click on the phrase they heard (i.e., the post-nominal order). If they selected the phrase with the English order, the button turned red and the trial was repeated. For each participant, 20 nouns were randomly sampled from the full 30-item lexicon; 10 of these were repeated, such that there were 30 total training trials (with 10 nouns reserved as new items for the test phase). At each trial, a random noun was paired with a random modifier.

\footnotetext{
${ }^{4}$ This experiment was preregistered: the hypothesis, predictions, methodology, and analysis were specified ahead of time in order to promote transparency and replicability. All exclusion criteria and statistical analyses reported here follow our pre-registered plan. Documentation can be found at https://osf.io/2wpbk/. All stimuli are available for download from the GitHub repository associated with the preregistration mentioned above.
} 
After training, participants were told they would move on to a test phase where they must translate from English into the new language. On each test trial, an English phrase was presented orthographically, and participants were instructed to chose the translation from a set of four. Testing consisted of 20 single- and 30 two-modifier trials (randomly intermixed). Single-modifier trials were similar to training trials. Participants might see an English phrase such as "green car" and then two buttons: one with the label "green car" and the other with the label "car green". These trials served to exclude off-task participants (i.e., those who erroneously chose the English rather than new language order). In two-modifier test trials, participants saw English phrases such as "this green car". There were four possible translations. Two of the translations were pre-nominal (e.g., "this green car" and "green this car"), and were therefore off-task. The other were post-nominal (e.g., "car this green" and "car green this"). Of the two post-nominal orders, one was isomorphic while the other followed the surface order of English modifiers. These critical trials allowed us to measure participants' isomorphism preferences, in a context where they have received no prior instruction on how to order two modifiers relative to one another in the new language.

\subsubsection{Participants}

We recruited 104 participants from the University of Edinburgh (mean age $=21.5 \pm 3.5$ years). These were mostly undergraduate students from the School of Philosophy, Psychology and Language Sciences. Participants were randomly assigned to one of the three conditions (Dem-Adj=32, Dem$\mathrm{Num}=38$, Num-Adj=34). Participants were run by native-English-speaking experimenters in soundattenuated booths at the University. All materials were in English.

Our preregistered analysis plan specified that participants who performed below $85 \%$ accuracy on single-modifier test trials would be excluded from analysis. However, no participants performed under this threshold, and all data was therefore included.

\subsection{Results}

Mean rates of post-nominal isomorphic choice on two-modifier test trials can be seen in Fig. 4. Per our preregistration, we ran standard logistic mixed-effects models (in R Bates, Maechler, Bolker, \& Walker 2014) to evaluate whether participants in each condition chose isomorphic orders reliably above chance level. However, these models did not converge, likely because of the very high number of isomorphic responses. We thus opted to implement the exact analysis reported in Culbertson and Adger (2014). That is, we used Bayesian mixed-effects logistic regression (R package MCMCglmm Hadfield 2010). $p$ values were estimated by Markov chain Monte Carlo sampling.

For each condition, we ran a model including an intercept and random factors for Participant and Item (the noun in the phrase), with the binary dependent variable "isomorphic". A weak informative prior on the random effects was included, as reported in Culbertson and Adger (2014). Participants chose post-nominal isomorphic orders reliably above chance level in all conditions (Dem-Adj: $M=$ 87.8\%, $\hat{\beta}=4.97, p<0.001$; Dem-Num: $M=68.9 \%, \hat{\beta}=3.51, p<0.01$; and Num-Adj: $M=76.6 \%, \hat{\beta}=4.35, p<0.001)$.

\subsection{Discussion}

To summarise, we found that English-speaking participants who were taught a new language with post-nominal modifiers consistently chose isomorphic orders of those modifiers despite no explicit 


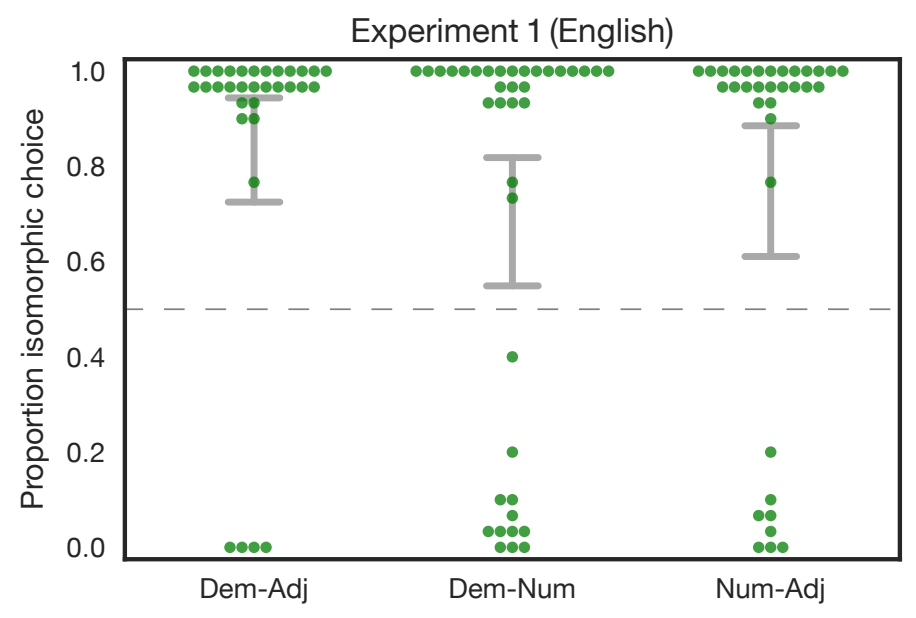

Figure 4: Rates of isomorphic order choice in two-modifier test trials by condition in Experiment 1. Error bars represent $95 \%$ confidence intervals on by-participant means; dots represent individual participant means.

training. This replicates the effect reported in Culbertson and Adger (2014) in a laboratory environment, and suggests a strong preference for isomorphic order (e.g., N Adj Dem) over L1 surface modifier order (e.g., N Dem Adj) in an artificial language learning task. As was the case in the original study, this result is consistent with the idea that English speakers represent and use an underlying structure of noun phrases in which adjectives form the tightest constituent with the noun, then numerals, then demonstratives. This could reflect knowledge due to experience with English, or it could reflect a universal representation which is hypothesised to explain the typological dominance of isomorphic orders. As pointed out by Culbertson and Adger (2014), if such results are to be explained by transfer from English, the nature of the transfer is crucial: If participants are biased by their English knowledge, it is in the abstract, hierarchical structure of their noun phrase representations, rather than by the surface order of the words.

\section{Experiment 2}

In Experiment 2, we teach a pre-nominal artificial language to speakers of a post-nominal language: Thai. The Thai noun phrase has the order N Adj Num Dem, as shown in (2).

(2) เก้าอี้ สีม่วง สอง ต้ว พวกนี้

kâw?îi š̌imûay sǒon tua p $\mathrm{p}^{\mathrm{h}}$ ak.níi

chair purple two clf pl-this

these two purple chairs

The Thai order is the most common typologically, and is the order English speakers inferred in Experiment 1 when exposed to a post-nominal version of English. Thai participants were exposed to an artificial version of Thai with pre-nominal modifiers (e.g., /níi kâw?îi/ rather than /kâw?îi níi/ - this chair rather than chair this). This allows us to test whether an isomorphism preference is 
found in a new speaker population whose native language has post-nominal modifiers. However, recall that the typological data suggest less flexibility in noun phrase order pre-nominally than postnominally: pre-nominal non-isomorphic orders are particularly rare. The accounts described above (Abels \& Neeleman 2012; Cinque 2005; Dryer 2018) therefore predict that because the new language Thai speakers are learning has pre-nominal modifiers, they should prefer isomorphic orderings more strongly than English speakers in Experiment 1.

\subsection{Methods}

Experiment 2 was identical to Experiment 1, except that Experiment 2 used Thai-language stimuli and a slightly modified adjective list (not all of the adjectives used in the English-language version map one-to-one with simple adjectives in Thai). ${ }^{5}$

\subsubsection{Stimuli}

The stimuli again consisted of 30 nouns, 10 adjectives, 10 numerals, and 4 demonstratives (นี้,/níi/ - "this"; น้น, /nán/ - "that"; พวกนี้, /pûakníi/ - "these"; พวกน้น, /pûaknán/ - "those"); they were recorded by a native female speaker of Thai from Bangkok in a sound-attenuated room. Note that the Thai noun phrase typically contains a classifier in the presence of a numeral (see (2) for an example). We chose to omit classifiers in order to make the Thai version of the experiment as similar to the English version as possible. ${ }^{6}$

\subsubsection{Procedure}

The procedure for Experiment 2 was identical to that of Experiment 1, except that the Thai participants were exposed to an artificial language with pre-nominal modifiers. Note this is the opposite of the new language presented to English-speaking participants in Experiment 1.

\subsubsection{Participants}

We recruited 106 participants from the Chulalongkorn University (mean age $=19.8 \pm 1.6$ years). These were mostly undergraduate students from the Faculty of Arts. Participants were randomly assigned to one of the three experimental conditions. Participants were run by native-Thai experimenters in a computer lab at the University. All materials were in Thai.

As for Experiment 1, participants who performed below 85\% accuracy on single-modifier test trials were to be excluded from analysis. One participant fell within this criterion and was excluded, resulting in the following number of participants per condition: Dem-Adj=33, Dem-Num=36, Num$\operatorname{Adj}=36$.

\footnotetext{
${ }^{5}$ As for Experiment 1, Experiment 2 was also preregistered, and all stimuli can be downloaded from https://osf.io/ $78 \mathrm{w} 2 \mathrm{a} /$.

${ }^{6}$ While definite noun phrases in Thai standardly feature a classifier morpheme (2), this can be omitted in informal speech (Piriyawiboon 2010). Omitting the classifier also allowed us to avoid deciding exactly what order it should be when creating a pre-nominal version of Thai.
} 


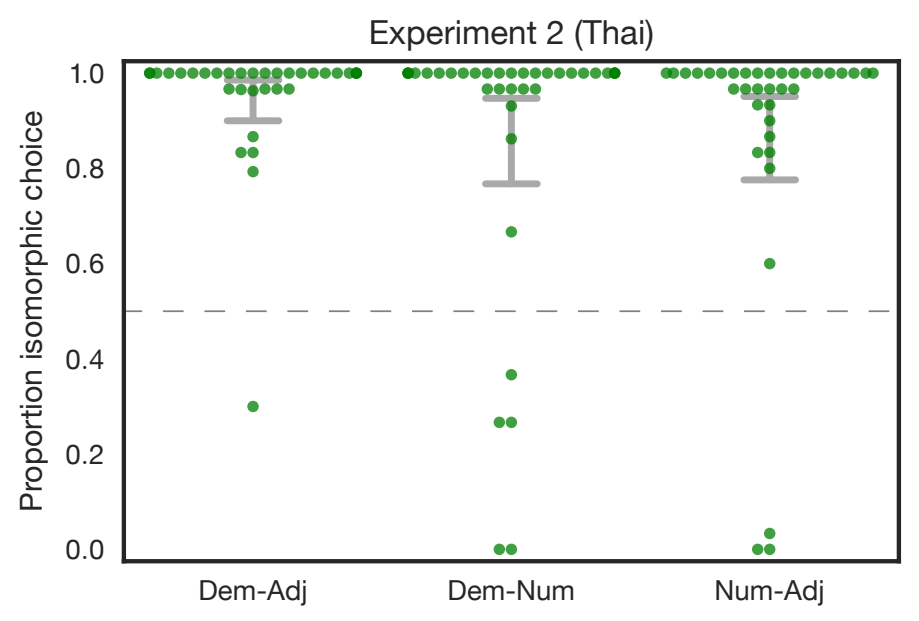

Figure 5: Rates of isomorphic order choice in two-modifier test trials by condition in Experiment 2. Error bars represent $95 \%$ confidence intervals on by-participant means; dots represent individual participant means.

\subsection{Results}

Mean rates of pre-nominal isomorphic choice on two-modifier test trials can be seen in Fig. 5. As for Experiment 1, we used Bayesian logistic mixed-effects models to analyse whether participants in each condition chose isomorphic orders reliably above chance level. For each condition, we ran a model including an intercept and random factors for Participant and Item (the noun in the phrase), with the binary dependent variable "isomorphic", and a weak informative prior on the random effects, following Culbertson and Adger (2014).

Participants chose pre-nominal isomorphic orders reliably above chance level in all conditions (Dem-Adj: $M=93.3 \%, \hat{\beta}=4.56, p<0.001$; Dem-Num: $M=79.4 \%, \hat{\beta}=3.75, p<0.01$; and Num-Adj: $M=84.0 \%, \hat{\beta}=3.82, p<0.001)$.

To test whether the preference for isomorphism was stronger for Thai-speaking participants (learning pre-nominal order) compared to English-speaking participants (learning post-nominal order), we compared the data from Experiments 1 and 2. Given that we did not predict a difference between conditions within each experiment (i.e., between different combinations of modifiers), we collapse these conditions for the analysis detailed here. We first ran a logistic mixed-effects model that included a fixed factor for Language (treatment coded) and random factors for Participant and Item (the noun in each phrase), with the binary dependent variable "isomorphic". The random factor for Item also included a random intercept for Language since each item (noun) was presented in both languages. This model was compared to one which excluded the fixed factor for Language. The model without Language was found to predict significantly less variance than the model including Language $\left(\beta=3.73, S E=1.00, \chi^{2}(1)=10.1, p<0.01\right)$. This significant effect of Language was driven by Thai-speaking participants choosing isomorphic orders on two-modifier test trials more often than English-speaking participants. 


\subsection{Discussion}

To summarise, in Experiment 2 we found that Thai speakers trained on a new language with prenominal modifiers showed a preference for isomorphic orders of those modifiers. This result replicates our findings with English speakers in Experiment 1, suggesting again that speakers use their knowledge of the underlying structural relations among modifiers in the noun phrase to infer word order in a new language. We further found that Thai speakers showed a stronger preference for isomorphism than the English speakers tested in Experiment 1, in line with previous accounts of noun phrase word order designed to explain an asymmetry between pre- and post-nominal non-isomorphic orders in the typology.

However, a stronger preference for isomorphism in pre-nominal systems is not the only potential explanation for the difference between populations we report here. By design, the native languages of our participants provide no evidence of isomorphism on the side of the noun tested (post-nominally in Experiment 1, pre-nominally in Experiment 2). However, our populations differ in the extent to which they have prior experience with another relevant language. Only four of the 104 English-speaking participants tested in Experiment 1 reported any knowledge of a post-nominal modifier language (specifically Igbo, Yoruba, or Shona). ${ }^{7}$ Therefore it is highly unlikely that the weaker isomorphism preference in Experiment 1 is due to English speaking participants' experience with other languages. On the other hand, all of the Thai-speaking participants tested in Experiment 2 reported knowledge of a pre-nominal modifier language, namely English. Although all instructions and materials were in Thai (presented by Thai-speaking experimenters), participants in Experiment 2 could in principle have mapped English structure onto the Thai-like artificial language. One way to see this is to look for a correlation between English knowledge and isomorphic choice in the experiment.

All participants answered a modified version of the Language Experience and Proficiency Questionnaire (LEAPQ) (Marian, Blumenfeld, \& Kaushanskaya 2007). We created a composite score of participants' reported English proficiency (the average of their self-reports in reading, writing, and speaking, all on a scale of 0 to 10), shown along with their proportion of isomorphic order choice in Fig. 6. Two things are evident from Fig. 6. First, there is very little variability in Thai participants' proportion of scope-isomorphic choice. This makes uncovering a correlation with English proficiency highly unlikely. Second, there is quite a bit of variability in participants' self-reported knowledge. This begs the question of just how much exposure to a language with pre-nominal modifiers is necessary to bias learners in a task like ours. Given the basic nature of the elements in question, and their systematic positioning in English (i.e., nearly categorically Dem Num Adj), it is possible that relatively little exposure is necessary. On the other hand, it is rare to encounter all three modifiers together; less than 3\% of noun phrases in the English corpora included in the Universal Dependencies Treebank (Nivre et al. 2017) include all three modifiers. In a post-test questionnaire 8 Thai participants explicitly referred to English order as influencing their responses, with the remainder providing explanations which overlapped largely with those of English participants (e.g., "swap words back to front", "put nouns at the end"8). We return to possible solutions to the issue of English knowledge in the General Discussion below.

\footnotetext{
${ }^{7}$ Many participants have experience with European languages like French, which have post-nominal adjectives. Crucially, though, these languages do not provide evidence for the relative ordering of modifiers post-nominally, since the other modifiers do not appear after the noun.

${ }^{8}$ English-speaking participants of course reported things like "put nouns at the beginning".
} 


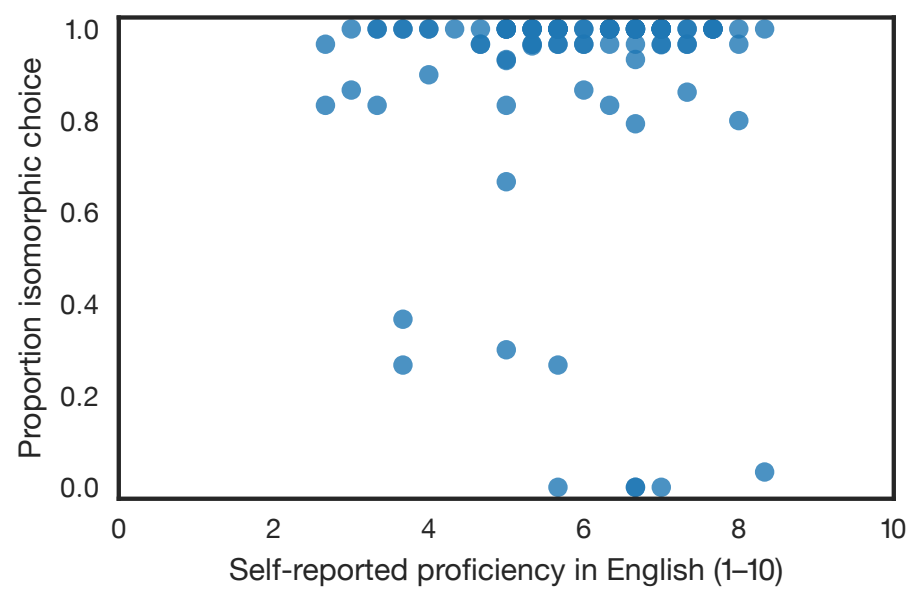

Figure 6: Proportion isomorphic order choice on two-modifier trials (all conditions combined) by self-reported English proficiency of participants in Experiment 2 (native speakers of Thai). Each dot represents a participant, where their English proficiency score was calculated by averaging their reported proficiency in English in reading, writing, and speaking on a scale of 0 to 10.

\section{General Discussion}

The aim of this paper was to build on previous typological and experimental evidence pointing to a possible preference for isomorphic orders in the noun phrase. These are orders in which the underlying constituency structure, or semantic scope relations, can be transparently recovered from linear order. Isomorphic orders are systematically more common among the world's languages compared to non-isomorphic orders, and English speakers have been shown to assume or infer them when taught an artificial language Culbertson and Adger (2014). The two artificial language learning experiments reported here demonstrate that this result is robust across learners from different linguistic populations (English speakers and Thai speakers). In both cases, we found a strong preference for participants to choose an isomorphic order, regardless of whether the artificial language had pre(Thai) or post-nominal (English) modifiers. Note that while these results may be explained by structural transfer from participants' L1, it is impossible to explain them based on L1 surface order transfer. Thus, if participants are transferring from their L1, they are doing so on the basis of abstract hierarchical structure. Further, we found that, in line with various syntactic theories (e.g., Abels \& Neeleman 2012; Cinque 2005; Dryer 2018), learners of a pre-nominal modifier language infer scope isomorphism (the Thai speakers in Experiment 2) at a higher rate than learners of a post-nominal modifier language (the English speakers in Experiment 1). This parallels the typological asymmetry between pre- and post-nominal modifier languages; while there are languages in which the default order of post-nominal modifiers is non-isomorphic, there are fewer (if indeed any) which exhibit non-scope-isomorphism of pre-nominal modifiers.

However, there two main limitations to our study. First, while Thai speakers' stronger preference for isomorphism is consistent with a constraint or bias against pre-nominal non-isomorphism, it could also be driven by their experience with English, a pre-nominal isomorphic language. Targeting a population without knowledge of English (or any other pre-nominal isomorphic language) is therefore needed to confirm our findings. While it is not possible to find university students in 
Thailand who have not been exposed to English (indeed the prevalence of English teaching worldwide makes this task difficult), in future work we will target young Thai-speaking children (prior to any major exposure to English). To do this will require substantial changes to the task (i.e., not using written stimuli, and likely not using re-ordered L1 words), and replication with English participants.

A second caveat concerns the hypothesis that a preference for isomorphism, independent of L1 experience, drives the typological prevalence of isomorphic orders. As in Culbertson and Adger (2014), both of the populations tested have prior experience with isomorphism in their language. To rule out an interpretation of our results based on abstract transfer rather than a universal isomorphism bias, we would have to test speakers of a non-isomorphic language. These are, of course, rare; however one possibility would be to test speakers of Kîtharaka, with its reported N Dem Num Adj order. ${ }^{9}$ If these speakers nevertheless infer an isomorphic order in a similar experiment, then we will have strong evidence for a universal isomorphism bias. This would also rule out the possibility that, rather than relying on the abstract structure of the noun phrase, participants in our task were using a conscious strategy of "flipping" or mirror-reversing their L1 order (e.g., as discussed in Culbertson \& Adger 2014). Both abstract transfer, and a flipping strategy would predict non-isomorphic responses from speakers of a language like Kîtharaka. Again, testing this population will require substantial changes to the task (e.g., not using written stimuli), and replication with English and Thai participants. We leave these follow-ups for future work.

To summarise, here we have provided further evidence in line with a hypothesised bias favouring noun phrase word orders that transparently reflect constituency structure, or semantic scope relations. We also have provided the first behavioural evidence consistent with a stronger isomorphism bias when modifiers are pre-nominal compared to post-nominal. These results remain to be confirmed with more ambitious experiments run in different linguistic populations.

\section{References}

Abels, K. \& Neeleman, A. (2012). Linear asymmetries and the LCA. Syntax, 15(1), 25-74.

Baker, M. C. (1985). The Mirror Principle and Morphosyntactic Explanation. Linguistic Inquiry, 16(3), 373-415.

Bates, D. M., Maechler, M., Bolker, B., \& Walker, S. (2014). lme4: Linear mixed-effects models using Eigen and S4.

Cinque, G. (2005). Deriving Greenberg's Universal 20 and Its Exceptions. Linguistic Inquiry, 36(3), $315-332$.

Culbertson, J. \& Adger, D. (2014). Language learners privilege structured meaning over surface frequency. Proceedings of the National Academy of Sciences of the United States of America, $111(16), 5842-5847$.

Cysouw, M. (2010). Dealing with diversity: Towards an explanation of NP-internal word order frequencies. Linguistic Typology, 14(2-3), 253-286.

Dryer, M. S. M. (2018). On the order of demonstrative, numeral, adjective and noun: An alternative to Cinque. Language, 1-16.

Evans, N. \& Levinson, S. C. (2009). The myth of language universals: language diversity and its importance for cognitive science. Behavioral and Brain Sciences, 32(5), 429-448.

\footnotetext{
${ }^{9}$ Although, ideally, we would need to target speakers without experience with any isomorphic language. This is nontrivial.
} 
Greenberg, J. H. (1963). Universals of language. Cambridge, Massachusetts: MIT Press.

Hadfield, J. D. (2010). MCMCglmm: MCMC Methods for Multi-Response GLMMs in R. Journal of Statistical Software, 33(2), 1-22.

Koopman, H. J. \& Szabolcsi, A. (2000). Verbal Complexes. Cambridge, Massachusetts: MIT Press.

Marian, V., Blumenfeld, H. K., \& Kaushanskaya, M. (2007). The Language Experience and Proficiency Questionnaire (LEAP-Q): Assessing language profiles in bilinguals and multilinguals. Journal of Speech Language and Hearing Research, 50(4), 940-967.

Muriungi, P. K. (2008). Phrasal movement inside Bantu verbs (Doctoral dissertation, University of Tromsø).

Nchare, A. L. (2012). The Grammar of Shupamem (Doctoral dissertation, New York University).

Nivre, J., Agić, Ž., Ahrenberg, L., Antonsen, L., Aranzabe, M. J., Asahara, M., ... Zhu, H. (2017). Universal Dependencies 2.1. LINDAT/CLARIN digital library at the Institute of Formal, Applied Linguistics (ÚFAL), Faculty of Mathematics, and Physics, Charles University.

Piantadosi, S. T. \& Gibson, E. (2014). Quantitative standards for absolute linguistic universals. Cognitive Science, 38(4), 736-756.

Piriyawiboon, N. (2010). Classifiers and Determiner-less Languages: The Case of Thai (Doctoral dissertation, University of Toronto).

Rice, K. (2000). Morpheme Order and Semantic Scope: Word Formation in Athapaskan Verb. Cambridge, UK.

Steddy, S. \& Samek-Lodovici, V. (2011). On the ungrammaticality of remnant movement in the derivation of Greenberg's Universal 20. Linguistic Inquiry, 42(3), 445-469.

Steedman, M. (2018). A formal universal of natural language grammar. 